\title{
NCI CTEP SDC Reproductive System Neoplasm, Male Category Terminology
}

National Cancer Institute

\section{Source}

National Cancer Institute. NCI CTEP SDC Reproductive System Neoplasm, Male

Category Terminology. NCl Thesaurus. Code C103027.

Reproductive System Neoplasm, Male is an NCl Cancer Therapeutic Evaluation Program (CTEP) Simplified Disease Classification (SDC) category used to organize cancer-related disease coding that harmonizes with and supports reporting based on the global standard Medical Dictionary for Drug Regulatory Reporting (MedDRA) terminology. 